\title{
Stoppage of Light Made Flexible by an Additional Control Field
}

\author{
G. S. Agarwal and Tarak Nath Dey \\ Physical Research Laboratory, Navrangpura \\ Ahmedabad 380 009, Gujarat, India
}

November 13, 2018

\begin{abstract}
We show how the application of a coupling field connecting the two lower metastable states of a Lambda system facilitates stoppage of light in a coherently driven Doppler broadened atomic medium via electromagnetically induced transparency.
\end{abstract}


In recent times one has learnt how the propagation of electromagnetic pulse could be manipulated by the use of the appropriate coherent fields $[1-13]$. This is because the propagation is dependent on the dispersive properties [14] of the medium and it is now understood how the dispersion of a medium can be manipulated by using control fields[1, 15]. As a matter of fact, regions of anomalous dispersion could be converted into regions of normal dispersion. It has been shown both theoretically and experimentally how the control fields could produce spatially varying refractive index profiles[16]. All these have remarkable bearing on the pulse propagation. Harris and coworkers $[1-2]$ first proposed how these control fields could be used to produce slow light. This was followed by a series of experiments in Bose condensates[3] as well as in hot atomic vapors $[4-6]$. These experiments demonstrated the production of ultraslow light. In a related development Wang et al. [7] demonstrated the production of superluminal propagation. They used the novel idea of using a bichromatic pump to produce a region of anomalous dispersion with negligible absorption or gain. A recent paper shows how the pulse propagation could be subjected to a new control parameter so that the same system can exhibit both sub- and superluminal propagation[13]. The experiments of Budker et al. [5] also exhibit superluminal behavior by the application of a suitable magnetic field. More recent experiments have demonstrated how the light could be stored in atomic coherences $[8,9]$ and how it can be retrieved at will. All these depend on the temporal dispersion of the medium and the experiments are interpreted in terms of dark polaritons [10].

In a recent work Kocharovskaya et al.[11] proposed how the spatial dispersion [17] can be used to stop light in a hot gas. They argued that the motion of atoms leads naturally to a refractive index or a susceptibility that is dependent on both the propagation vector and frequency. Explicit calculation for a $\Lambda$ system shows that the stoppage of light occurs when the control fields is suitably detuned from the atomic transition and when the central frequency of the probe pulse satisfies the two-photon resonance condition. The mechanism proposed by Kocharovskaya et al.[11] is quite different from the one of Ref. [10], as it does not require the switching off and switching on of the control field.

In the present Communication we show how we can make the stoppage of light flexible by using another control field. We consider a $\Lambda$ system as shown in Fig. 1(a). We apply two control fields one on the transition $|1\rangle \leftrightarrow|2\rangle$ and the other on the transition $|2\rangle \leftrightarrow|3\rangle$. The probe pulse acts on the transition $|1\rangle \leftrightarrow$ $|3\rangle$. The transition $|2\rangle \leftrightarrow|3\rangle$ is generally electric dipole forbidden transition. The states $|2\rangle$ and $|3\rangle$ are metastable states. We calculate the group velocity of the pulse for different strengths of the two control fields. We demonstrate existence of very wide region of parameters where the stoppage of light can be achieved while maintaining regions of very low absorption.

In general for a spatially dispersive medium[17] the response of the medium can be expressed by a susceptibility $\chi(\mathrm{k}, \omega)$ that is dependent on the propagation vector and frequency. Further the allowed wavevectors are given by the 
dispersion relation

$$
\mathrm{k}^{2}=\frac{\omega^{2}}{c^{2}}[1+4 \pi \chi(\mathrm{k}, \omega)]
$$

It is well known that the above dispersion relation can lead to many real solutions for $\mathrm{k}$ for a fixed $\omega$ and thus one has the possibility of additional waves in a spatially dispersive medium. In this paper we however consider only the case when $|\chi|$ is much smaller than one. In this case we would basically have one wave. A simple analysis now shows that the group velocity is given by

$$
v_{g}=\left[\frac{c\left(1-2 \pi \mathrm{k} \frac{\partial \chi}{\partial \mathrm{k}}\right)}{1+2 \pi \omega \frac{\partial \chi}{\partial \omega}}\right] .
$$

This formula assumes weak spatial as well as temporal dispersion. Besides we assume that absorption is negligible. In a gas of atoms, the $\chi(\mathrm{k}, \omega)$ is to be replaced by the average values $\chi(\omega-\mathrm{k} v)$ over the distribution of velocities. Then the expression for the group velocity becomes

$$
v_{g}=\operatorname{Re}\left[\frac{c\left(1+2 \pi \mathrm{k}\left\langle v \frac{\partial \chi}{\partial \omega}\right\rangle\right)}{1+2 \pi \omega\left\langle\frac{\partial \chi}{\partial \omega}\right\rangle}\right] .
$$

Note that

$$
\left\langle v \frac{\partial \chi}{\partial \omega}\right\rangle \neq\langle v\rangle\left\langle\frac{\partial \chi}{\partial \omega}\right\rangle \neq 0
$$

and hence the stoppage of light takes place if the numerator in Eq. (3) vanishes. The susceptibility $\chi(\omega)$ will depend strongly on the intensities and the frequencies of the two control fields. The susceptibility $\chi(\omega)$ is obtained by solving the density matrix equations for the $\Lambda$ system of Fig. 1(a), i.e, by calculating the density matrix element $\rho_{13}$ to first order in the applied optical field [18] on the transition $|1\rangle \leftrightarrow|3\rangle$ but to all orders in the two control fields. We define all fields as

$$
\vec{E}(z, t)=\overrightarrow{\mathcal{E}}(z) e^{-i(\omega t+\phi)}+\text { c.c. }
$$

By making a unitary transformation from the density matrix $\rho$ to $\sigma$ via

$$
\rho_{12}=\sigma_{12} e^{-i\left(\omega_{2} t+\phi_{2}\right)}, \rho_{13}=\sigma_{13} e^{-i\left(\omega_{2}+\omega_{3}\right) t-i\left(\phi_{2}+\phi_{3}\right)}, \rho_{23}=\sigma_{23} e^{-i\left(\omega_{3} t+\phi_{3}\right)},
$$

we have the relevant density matrix equations

$$
\begin{aligned}
\dot{\sigma}_{11} & =i G \sigma_{21}+i g e^{-i\left(\Delta_{4} t+\delta \phi\right)} \sigma_{31}-i G^{*} \sigma_{12}-i g^{*} e^{i\left(\Delta_{4} t+\delta \phi\right)} \sigma_{13}-2\left(\gamma_{1}+\gamma_{2}\right) \sigma_{11} \\
\dot{\sigma}_{22} & =i G^{*} \sigma_{12}+i \Omega \sigma_{32}-i G \sigma_{21}-i \Omega^{*} \sigma_{23}+2 \gamma_{2} \sigma_{11} \\
\dot{\sigma}_{12} & =-\left[\gamma_{1}+\gamma_{2}+\Gamma_{12}-i \Delta_{2}\right] \sigma_{12}+i G \sigma_{22}+i g e^{-i\left(\Delta_{4} t+\delta \phi\right)} \sigma_{32} \\
& -i G \sigma_{11}-i \Omega^{*} \sigma_{13} \\
\dot{\sigma}_{13} & =-\left[\gamma_{1}+\gamma_{2}+\Gamma_{13}-i\left(\Delta_{2}+\Delta_{3}\right)\right] \sigma_{13}+i G \sigma_{23}+i g e^{-i\left(\Delta_{4} t+\delta \phi\right)} \sigma_{33} \\
& -i g e^{-i\left(\Delta_{4} t+\delta \phi\right)} \sigma_{11}-i \Omega \sigma_{12} \\
\dot{\sigma}_{23} & =-\left(\Gamma_{23}-i \Delta_{3}\right) \sigma_{23}+i G^{*} \sigma_{13}+i \Omega \sigma_{33}-i g e^{-i\left(\Delta_{4} t+\delta \phi\right)} \sigma_{21}-i \Omega \sigma_{22}
\end{aligned}
$$


Here $\Gamma$ 's give collisional dephasing terms; $\gamma$ 's give the radiative decay of the state $|1\rangle ; \Delta_{i}$ 's are the detunings

$$
\Delta_{1}=\omega_{1}-\omega_{13}, \quad \Delta_{2}=\omega_{2}-\omega_{12}, \quad \Delta_{3}=\omega_{3}-\omega_{23}, \quad \Delta_{4}=\omega_{1}-\omega_{2}-\omega_{3},
$$

and the relative phase difference of the probe field to the sum of the pump and additional control field is

$$
\delta \phi=\phi_{1}-\phi_{2}-\phi_{3} .
$$

The coupling constants $2 g$ and $2 G$ are the Rabi frequencies of the probe and the optical control field, while $g=\vec{d}_{13} \cdot \overrightarrow{\mathcal{E}}_{p} / \hbar$ and $G=\vec{d}_{12} \cdot \overrightarrow{\mathcal{E}}_{c} / \hbar$. The parameter $\Omega$ characterizes the coupling between two lower levels. We will refer to this field as LL coupling field. The susceptibility $\chi$ can be obtained by considering the steady state solution of (7) to first order in the probe field on the transition $|1\rangle \leftrightarrow|3\rangle$. For this purpose we assume $\gamma_{1}=\gamma_{2}=\gamma$ and write the solution as

$$
\sigma=\sigma^{0}+\frac{g}{\gamma} e^{-i\left(\Delta_{4} t+\delta \phi\right)} \sigma^{+}+\frac{g^{*}}{\gamma} e^{i\left(\Delta_{4} t+\delta \phi\right)} \sigma^{-}+\ldots \ldots
$$

On combining Eqs.(6) and (10), we note that to first order $\rho_{13}(t) \equiv(g / \gamma) e^{-i\left(\omega_{1} t+\phi_{1}\right)}$ $\sigma_{13}^{+}$. Thus 13 element of $\sigma^{+}$will give the susceptibility at the frequency $\omega_{1}$ which now can be expressed in the form

$$
\chi\left(\omega_{1}\right)=\frac{n\left|\vec{d}_{13}\right|^{2}}{\hbar \gamma} \sigma_{13}^{+},
$$

where $n$ is the density of the atoms. The phase dependence of different fields does not appear in the susceptibility . Note that the zeroth order contribution in Eqs.(10) can result in phase dependent components. However for the range of parameters used in this paper, the zeroth order term is so small that it can be ignored.

To obtain the probe response in a Doppler-broadened medium $\sigma_{13}^{+}$should be averaged over the Maxwell-Boltzmann velocity distribution of the moving atoms. For a single atom, moving with a velocity $v$ along the $z$ axis, the probe frequency $\omega_{1}(v)$ and frequencies $\omega_{2}(v), \omega_{3}(v)$ of the two control fields as seen by the atom are given by

$$
\omega_{1}(v)=\omega_{1}-\mathrm{k}_{1} v, \quad \omega_{2}(v)=\omega_{2}-\mathrm{k}_{2} v, \quad \omega_{3}(v)=\omega_{3}-\mathrm{k}_{3} v .
$$

Thus susceptibilities for moving atoms are obtained by using the replacement (12) in the solution of Eqs. (7). Note that the velocity dependence of $\omega_{3}$ is insignificant and can be dropped. For simplicity we can also set $k_{1} \approx k_{2}$. These susceptibilities are to be averaged over the Maxwell-Boltzmann distribution for the atomic velocities, defined by

$$
\mathrm{P}\left(k_{1} v\right) d\left(k_{1} v\right)=\frac{1}{\sqrt{2 \pi \mathrm{D}^{2}}} e^{-\left(k_{1} v\right)^{2} / 2 \mathrm{D}^{2}} d\left(k_{1} v\right), \quad \mathrm{D}=\sqrt{K_{B} T \omega_{1}^{2} / M c^{2}} .
$$


We next give the results of our calculations for the model systems shown in the Fig. 1(a). We show a number of numerical results in Figs. 1, 2 and 3. In Fig. 1(b) and Fig. 1(c) we show the behavior of the susceptibility as a function of the detuning of the probe when the control field $\omega_{2}$ is detuned, $\Delta_{2}=-50 \gamma$. It is clear from the Fig. 1(b) that with increase of the microwave field intensity results in decrease of the probe absorption in presence of collisional dephasing. At two-photon resonance condition i.e., $\Delta_{1}=\Delta_{2}=-50 \gamma$, the absorption of the probe is very small. Therefore the transparency window is obtained at $\Delta_{1}=\Delta_{2}$. Note that the transparency dip that appears in the absorption spectrum has finite bandwidth and its width depends on coupling fields $G$ and $\Omega$. The probe pulse spectrum should be contained within this finite bandwidth. However, the transparency dip is a accompanied by a steep variation of $\langle\operatorname{Re}[\chi]\rangle$ with probe detuning. We find that if two control fields are suitably detuned then the light can be stopped. We show in the Fig. 2(a) how the group velocity $v_{g}$ as defined in Eqs. (3), changes from negative values to large positive values as the intensity of the LL coupling field is increased. The group velocity $v_{g}$ become zero at the value of $\Omega=1741 \times 10^{-6} \gamma$ when the control field $G$ is out of one-photon resonance but satisfies the two photon resonance condition $\left(\Delta_{1}=\Delta_{2}=-50 \gamma\right)$. Note that for ${ }^{87} \mathrm{Rb}$, a Rabi frequency of $10^{-6} \gamma$ implies a magnetic field of the order of $.993 \mu \mathrm{G}$. The slope of $\langle\operatorname{Re}[\chi]\rangle$ with respect to central frequency of the probe pulse depends on the intensity of the two control fields and density of atoms. The group velocity becomes zero [Fig. 2(b)] as the numerator in Eq. (3) changes sign when the LL coupling field is increased. The Fig. 2(c) gives the group velocity in the absence of spatial dispersion. A comparison of the Figs. 2(a) and 2(c) shows the important role played by spatial dispersion. Further we notice from the Fig. 2(d) that at resonance condition $\left(\Delta_{1}=\Delta_{2}=0\right)$ light cannot be stopped. In order to understand how the application of the microwave field leads to the stoppage of light, we show in the Fig. 3 the behavior of the Doppler average of $\left\langle\partial \chi / \partial \omega_{1}\right\rangle$ and $\left\langle 1-2 \pi \mathrm{k}_{1} \frac{\partial \chi}{\partial \mathrm{k}_{1}}\right\rangle$ as a function of $\omega_{1}$. The latter quantity crosses zero which results in the stoppage of light. The Figs. 2 and 3 show how the application of the microwave field changes all the physical quantities. At a more fundamental level the microwave field and pump field together produce new dressed states of the system. Such dressed states determine the response of the system to the applied probe field. However all these can be understood easily only for a homogeneously broadened system.

Thus in conclusion we have demonstrated how the application of an LL coupling field in the $\Lambda$ system helps one to change the group velocity of the pulse inside the medium from a negative to positive value, and thereby helps in stopping light. Thus for a suitable detuning of the pump and probe fields, one can stop light by just changing the intensity of the LL coupling field.

G. S. Agarwal thanks M. O. Scully for useful discussions on the subject of pulse propagation. 


\section{References}

[1] Harris, S. E., Field, J. E., and Kasapi, A., 1992, Phys. Rev. A, 46, R29 .

[2] Kasapi, A., Jain, M., Yin, G. Y., and Harris, S.E., 1995, Phys. Rev. Lett., 74, 2447.

[3] Hau, L. V., Harris, S. E., Dutton, Z., and Behroozi, C. H., 1999, Nature, $\mathbf{3 9 7}, 594$.

[4] Kash, M. M., Sautenkov, V. A., Zibrov, A. S., Hollberg, L., Welch, G. R., Lukin, M. D., Rostovtsev, Y., Fry, E. S., and Scully, M. O., 1999, Phys. Rev. Lett., 82, 5229; Mastko, A. B., Kocharovskaya, O., Rostovtsev, Y., Zibrov, A. S., Scully, M. O., 2001, Adv. Mol. Phys., 46, 191.

[5] Budker, D., Kimball, D. F., Rochester, S. M., and Yashchuk, V. V., 1999, Phys. Rev. Lett., 83, 1767.

[6] Schmidt, O., Wynands, R., Hussein, Z., and Meschede, D., 1996, Phys. Rev. A, 53, R27.

[7] Wang, L. J., Kuzmich, A., and Dogariu, A., 2000, Nature, 406, 277; Dogariu, A., Kuzmich, A., and Wang, L. J., 2001, Phys. Rev. A, 63, 053806.

[8] Liu, C., Dutton, Z., Behroozi, C. H., and Hau, L. V., 2001, Nature, 409, 490.

[9] Phillips, D. E., Fleischhauer, A., Mair, A., Walsworth, R. L., and Lukin, M. D., 2001, Phys. Rev. Lett., 86, 783.

[10] Fleischhauer, M., and Lukin, M. D., 2000, Phys. Rev. Lett., 84, 5094; Nonadiabatic effects in storage of light are discussed by - Matsko, A. B., Rostovsev, Y. V., Kocharovskaya,O., Zibrov, A. S., and Scully, M. O., 2001, Phys. Rev. A, 64, 043809.

[11] Kocharovskaya, O., Rostovtsev, Y., and Scully, M. O., 2001, Phys. Rev. Lett., 86, 628 .

[12] Bortman-Arbiv, D., Wilson-Gordon, A. D., and Friedmann, H., 2001, Phys. Rev. A, 63, 043818.

[13] Agarwal, G. S., Dey T. N., and Menon, S., 2001, Phys. Rev. A, 64, 053809.

[14] Garrett, C. G. B., and McCumber, D. E., 1970, Phys. Rev. A, 1, 305.

[15] Tewari, S. P., and Agarwal, G. S., Phys. Rev. Lett., 1986, 56, 1811. 
[16] Kapoor, R., and Agarwal, G. S., 2000, Phys. Rev. A, 61, 053818; Moseley, R. R., Shepherd, S., Fulton, D. J., Sinclair, B. D., and Dunn, M. H., 1995, Phys. Rev. Lett., 74, 670; Moseley, R. R., Shepherd, S., Fulton, D. J., Sinclair, B. D., and Dunn, M. H., 1996, Phys. Rev. A, 53, 408; Ling, H. Y., Li, Y. Q., and Xiao, M., 1998, Phys. Rev. A, 57, 1338.

[17] Agranovich, V. M., and Ginzburg, V. L., 1964, Spatial dispersion in crystal optics and the theory of excitons, ( London, Interscience Publishers).

[18] It is not necessary to calculate upto first order in probe; exact calculation is possible. However one studies concepts like group velocity in a linear medium and therefore we treat probe to first order so that the medium is linear as far as probe is concerned. 


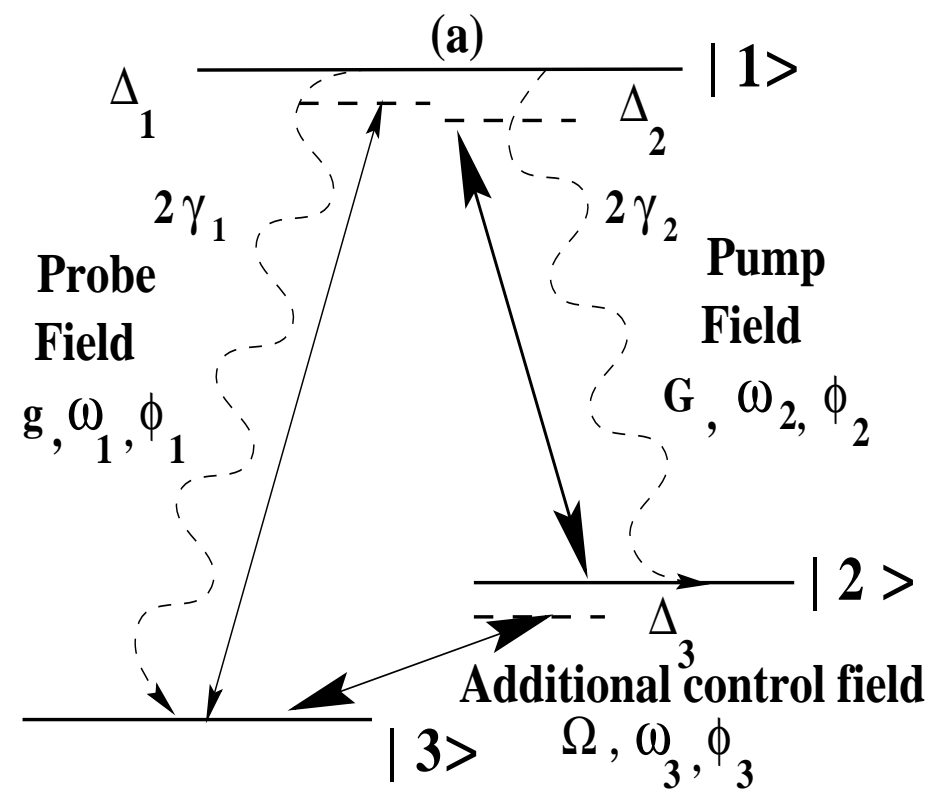


(b)



(c)



Figure 1: (a) Schematic diagram of three level $\Lambda$-system; the probe pulse is applied on the transition $|1\rangle \leftrightarrow|3\rangle$; other fields are cw. (b) and (c) The imaginary and real parts of susceptibility $\langle[\chi]\rangle$ at the probe frequency $\omega_{1}$ in the presence of control field $G$ and LL coupling field $\Omega$. Detuning $\Delta_{2}$ of the control field is chosen as $-50 \gamma$. The common parameters of the above three graphs for ${ }^{87} \mathrm{Rb}$ vapor are chosen as: Doppler width parameter $\mathrm{D}=1.33 \times 10^{9} \mathrm{rad} / \mathrm{sec}$, density $n=10^{12}$ atoms $/ \mathrm{cc}, G=0.3 \gamma, \Delta_{3}=0, \Gamma_{12}=\Gamma_{13}=0, \gamma=3 \pi \times 10^{6} \mathrm{rad} / \mathrm{sec}$. 
(a)



(c)

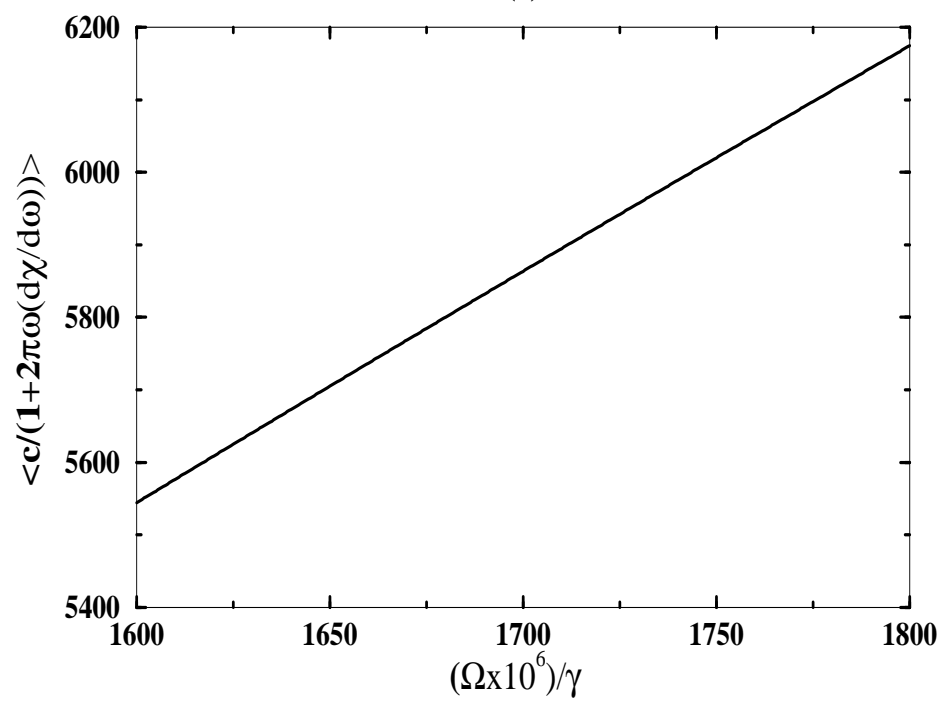

(b)



(d)

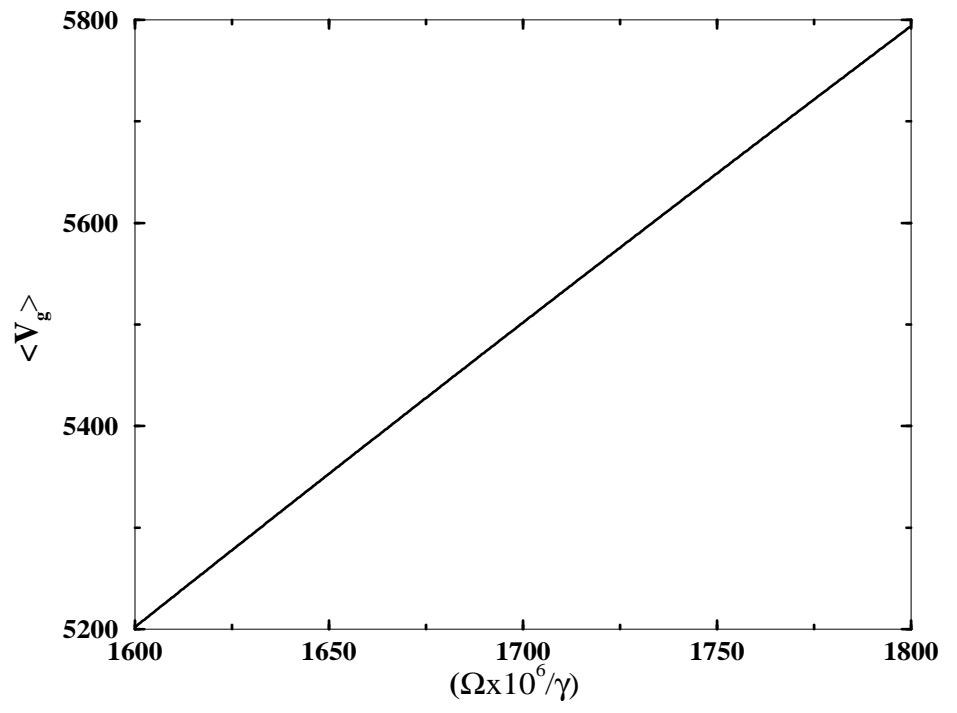

Figure 2: (a) shows variation of group velocity, in $\mathrm{cm}$ per sec, of Eq.(3) with the strength $\Omega$ of the LL coupling field. The group velocity becomes zero because the numerator in Eq. (3) becomes zero as shown in the Fig. 2(b). The Fig. 2(c) gives the behavior of the group velocity if the spatial dispersion of the susceptibility were ignored. The common parameters of the above three graphs for ${ }^{87} \mathrm{Rb}$ vapor are chosen as: Doppler width parameter $\mathrm{D}=1.33 \times 10^{9} \mathrm{rad} / \mathrm{sec}$, density $n=10^{12}$ atoms $/ \mathrm{cc}, G=$ $0.3 \gamma, \Delta_{3}=0, \Gamma_{12}=\Gamma_{13}=0, \Gamma_{23}=0.001 \gamma, \gamma=3 \pi \times 10^{6} \mathrm{rad} / \mathrm{sec}, \Delta_{1}=\Delta_{2}=-50 \gamma$. For comparison we also show in the Fig. 2(d) the result for $\Delta_{1}=\Delta_{2}=0$. 
(a)

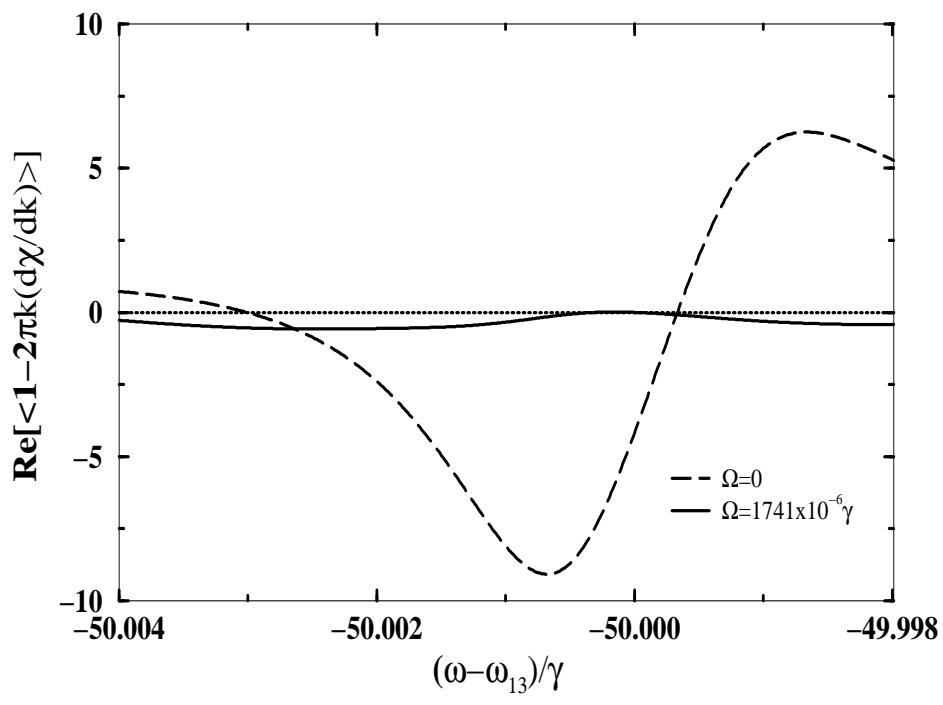

(b)



Figure 3: (a) shows the variation of the numerator in Eq.(2) with the probe detuning taking Doppler effect into account. The Fig. 3(b) gives the slope of the susceptibility. The common parameters of the above two graphs for ${ }^{87} \mathrm{Rb}$ vapor are chosen as: Doppler width parameter $\mathrm{D}=1.33 \times 10^{9} \mathrm{rad} / \mathrm{sec}$, density $n=10^{12}$ atoms $/ \mathrm{cc}, G=0.3 \gamma, \Delta_{3}=0, \Delta_{2}=-50 \gamma \Gamma_{12}=\Gamma_{13}=0, \Gamma_{23}=0.001 \gamma$, $\gamma=3 \pi \times 10^{6} \mathrm{rad} / \mathrm{sec}$. 